\title{
Advantages of expanded universal carrier screening: what is at stake?
}

\begin{abstract}
Sanne van der Hout ${ }^{\star, 1}$, Kim CA Holtkamp ${ }^{2}$, Lidewij Henneman ${ }^{2}$, Guido de Wert ${ }^{1}$ and Wybo J Dondorp ${ }^{1}$
Expanded universal carrier screening (EUCS) entails a twofold expansion of long-standing (preconception) carrier screening programmes: it not only allows the simultaneous screening of a large list of diseases ('expanded'), but also refers to a pan-ethnic screening offer ('universal'). Advocates mention three main moral advantages of EUCS as compared with traditional (targeted and/or ancestry-based) forms of carrier screening: EUCS will (1) maximise opportunities for autonomous reproductive choice by informing prospective parents about a much wider array of reproductive risks; (2) provide equity of access to carrier testing services; (3) reduce the risk of stigmatisation. This empirical ethics study aims to widen this account and provide a balanced picture of the potential pros and cons of EUCS. Semi-structured interviews were conducted with 17 health (policy) professionals and representatives of patient organisations about their views on carrier screening including a possible EUCS scenario. Stakeholders acknowledged the potential benefits of EUCS, but also expressed five main moral concerns: (1) Does EUCS respond to an urgent problem or population need? (2) Is it possible to offer couples both understandable and sufficient information about EUCS? (3) How will societal views on 'reproductive responsibility' change as a result of EUCS? (4) Will EUCS lead to a lower level of care for high-risk populations? (5) Will EUCS reinforce disability-based stigmatisation? While having the potential to overcome some moral limits inherent in traditional carrier screening, EUCS comes with moral challenges of its own. More research is needed to (further) anticipate the ethical and practical consequences of EUCS.
\end{abstract}

European Journal of Human Genetics (2017) 25, 17-21; doi:10.1038/ejhg.2016.125; published online 28 September 2016

\section{INTRODUCTION}

Preconception carrier screening (PCS) for single-gene autosomal recessive disorders has been offered since the 1970s. The first successful carrier screening initiatives addressed individuals from specific ethnic groups for diseases known to be more prevalent in these populations. This type of screening has been referred to as 'ancestry-based' or 'ethnicity-based' carrier screening. ${ }^{1,2}$ A well-known example is beta-thalassemia carrier screening in several high-risk populations in the Mediterranean region. ${ }^{3}$ Another example regards the Ashkenazi Jewish population, which is at increased risk for several recessively inherited disorders. ${ }^{4}$ Initially targeted at Tay-Sachs disease, screening programmes for the Jewish population have expanded since, with panels now including up to 38 diseases. ${ }^{2}$ A more recent development is the promotion of universal approaches that offer screening to all individuals regardless of race or ethnicity, for instance carrier screening for cystic fibrosis (CF). Originally, US screening guidelines for CF addressed Caucasians and Ashkenazi Jewish populations. However, as 'it is becoming increasingly difficult to assign a single ethnicity to affected individuals', the American College of Obstetricians and Gynecologists has been recommending CF carrier screening for all couples since $2011 .^{5}$ Carrier screening can be offered either during or before pregnancy. PCS has the advantage of allowing a larger range of reproductive choices than only prenatal diagnosis followed by a possible termination of pregnancy. ${ }^{6}$

The emergence of new genetic technologies has given universal carrier screening a new incentive: high-throughput platforms and sequencing approaches allow for efficient screening of a large number of diseases at a faster turnaround time and lower costs. Such 'expanded carrier screening' (ECS $)^{2,7-9}$ provides information about many more disorders than recommended in current screening guidelines: panels often include more than 100 recessive diseases, most of which are rare. The possibility to assess a large number of diseasecausing mutations with a single screening panel is expected to provide valuable information for people who do not belong to the identified high-risk groups. When people are offered screening for only one or a few genetic conditions, they often view their individual risk for an affected pregnancy as being rather low. The utility of screening is likely to be far higher when risk is perceived from the perspective of the combined risk for a wide range of diseases. ${ }^{10}$

The availability of broad screening panels thus seems to encourage the implementation of a population-wide screening offer. The

\begin{tabular}{|c|c|c|}
\hline & Targeted & Expanded \\
\hline $\begin{array}{r}\text { Ancestry- } \\
\text { based }\end{array}$ & $\begin{array}{l}\text { 1. Beta-thalassemia } \\
\text { (Mediterranean region) }\end{array}$ & $\begin{array}{l}\text { 2. Ashkenazi Jewish } \\
\text { screening panels }\end{array}$ \\
\hline Universal & $\begin{array}{l}\text { 3. Cystic Fibrosis } \\
\text { (US population) }\end{array}$ & $\begin{array}{l}\text { 4. Broad-scope screening } \\
\text { panels for a general } \\
\text { population }\end{array}$ \\
\hline
\end{tabular}

Figure 1 Schematic overview of the different types of (preconception) carrier screening, including examples.

\footnotetext{
${ }^{1}$ Department of Health, Ethics and Society, Research Schools CAPHRI and GROW, Maastricht University, Maastricht, The Netherlands; ${ }^{2}$ Department of Clinical Genetics, Section of Community Genetics, EMGO Institute for Health and Care Research, VU University Medical Center, Amsterdam, The Netherlands

${ }^{*}$ Correspondence: Dr S van der Hout, Department of Health, Ethics and Society, Research Schools CAPHRI and GROW, Maastricht University, PO Box 616,6200 MD Maastricht, The Netherlands. Tel: +31 4338 82285; E-mail: s.vanderhout@maastrichtuniversity.nl

Received 7 March 2016; revised 30 June 2016; accepted 24 August 2016; published online 28 September 2016
} 
incentive also appears to work the other way around: population-wide screening makes more sense if people are tested for a large list of diseases. Figure 1 gives a schematic overview of the different variants of (preconception) carrier screening that are currently offered. To emphasise that ECS implies a twofold expansion, we will henceforth use the term 'expanded universal carrier screening' (EUCS).

EUCS has been offered by commercial laboratories in North America, Australia and Europe for almost a decade. ${ }^{11}$ More recently, it has started to attract the attention of the non-profit health sector. ${ }^{12}$ The benefits of EUCS have been increasingly acknowledged in reports, position statements and other publications. ${ }^{7,13}$ Moreover, some of these publications specifically indicate a preference for EUCS over traditional (targeted and/or ancestry-based) screening protocols. ${ }^{2,10,14}$ Advocates mention three main moral advantages of this new screening proposition. First of all, EUCS would better serve the aim of reproductive screening, which is to enhance opportunities for autonomous reproductive choice. It contributes to this very aim by providing information about a much wider array of reproductive risks than traditional (targeted and/or ancestry-based) forms of carrier screening. So much, at least, is the claim: whereas 'screening for individual conditions limits the amount of accessible genetic information for participants', ${ }^{7}$ EUCS provides (prospective) parents with much more genetic information that might be relevant for making reproductive decisions. $^{2}$

Second, EUCS would ensure that this genetic information will be available to all those who may benefit from it. ${ }^{13}$ In many countries, carrier screening is currently available to only selected parts of the population. By offering all individuals screening for the same set of conditions, EUCS will result in an equitable application of genomic technology'. ${ }^{14}$ This seems especially important as in today's ethnically diverse populations it is very difficult to define who exactly is at risk of being a carrier of a severe genetic condition.

Finally, EUCS would 'potentially reduce [...] the risk of stigmatisation of ethnic groups'. ${ }^{15}$ Stigmatisation is the result of labelling a typically easily identifiable - group with negative social characteristics. In the 1970s, 'social stigma' was frequent among carriers of sickle cell disease. In Greece, 'carriers were isolated, socially ostracized, and considered [...] undesirable marriage partners'. ${ }^{16}$ Encouragingly, more recent studies do not reveal predominant feelings of social stigma among carriers. ${ }^{17}$ There have also been concerns that carriers may suffer from 'self-stigma' linked to a poorer health perception. ${ }^{18}$ Axworthy et $a l^{19}$ reported that informing people that they were CF carriers had a negative effect on how they perceived their health. Other studies, however, did not confirm this. ${ }^{20}$ Suggesting (rightly or wrongly) that concerns about possible stigmatisation are not entirely hypothetical, proponents of EUCS have argued that this approach would have the advantage of further reducing this risk. By addressing the whole population and looking for a wide array of disorders, it will show that being a carrier is not at all exceptional. ${ }^{21}$

Advocates of EUCS present this new form of screening as a beneficial proposition beyond questioning. However, it can be asked if the advantages of EUCS are as unambiguous as suggested by its proponents. By exploring the views of stakeholders in professions or roles that in various ways would be involved in the implementation of EUCS in the health sector, we aim to provide a balanced and nuanced picture of the possible pros and cons of EUCS.

\section{MATERIALS AND METHODS}

Semi-structured interviews were conducted by two researchers (KH and EV) with 17 expert stakeholders (health (policy) professionals and representatives of patient organisations) about their views on barriers to and facilitators of
Table 1 Professional backgrounds of respondents and interview settings

\begin{tabular}{lll}
\hline Respondent no. & \multicolumn{1}{c}{ Respondent background } & Interviewed \\
\hline$\# 1$ & Midwife & Face-to-face \\
$\# 2$ & Researcher (midwife) & Face-to-face \\
$\# 3$ & Paediatrician & Face-to-face \\
$\# 4$ & Staff member of patient organisation & Face-to-face \\
$\# 5$ & Bio-ethicist & Skype \\
$\# 6$ & Clinical geneticist & Face-to-face \\
$\# 7$ & Molecular geneticist & Face-to-face \\
$\# 8$ & Staff member of patient organisation & Face-to-face \\
$\# 9$ & Staff member of patient organisation & Face-to-face \\
$\# 10$ & Scientific researcher & Face-to-face \\
$\# 11$ & Youth health care physician & Telephone \\
$\# 12$ & Midwife & Telephone \\
$\# 13$ & General practitioner & Telephone \\
$\# 14$ & Clinical geneticist & Telephone \\
$\# 15$ & Midwife & Face-to-face \\
$\# 16$ & General practitioner & Face-to-face \\
$\# 17$ & Coordinator at Ministry of Health & Face-to-face \\
\hline
\end{tabular}

implementing carrier screening. Stakeholders were selected because of their (potential) involvement in the implementation of carrier screening in the Dutch health sector. The interviews took place between October 2014 and August 2015 and lasted $45 \mathrm{~min}$ on average. The interviews were audiotaped and transcribed verbatim afterwards. Table 1 provides information about the professional backgrounds of the respondents involved in this study, as well as the setting of the interviews.

The results presented in this paper concentrate on one particular topic included in the interview questionnaire: the emerging shift from ancestry-based carrier screening to EUCS. The interview questions touching on this topic were: (a) To whom should carrier screening be offered? (b) What types of disorders should be included in the screening panel? Other results from the interview study will be presented elsewhere (Holtkamp et $a l$, in preparation). Respondents were not explicitly asked to express their views on the moral benefits of EUCS as presented by its advocates. Our motivation to nonetheless concentrate on this topic was that in analysing the interview data, we found that respondents explicitly commented on all three supposed advantages.

Initially, the transcripts were read and reread to identify and index themes and categories. Through a process of constant comparison, the data were classified and clustered into key themes and subcategories. ${ }^{22}$ Data analysis continued until no new themes emerged from the interviews. Five interviews were independently coded by two researchers (SvdH, KH). Coding was compared for reliability and discrepancies were discussed until agreement was reached. All remaining interviews were coded by SvdH and discussed by the whole research team. Representative quotes were selected to illustrate the views of stakeholders as regards the desirability of EUCS. All quotes were translated into English while preserving the verbatim character of the original statements. To secure the anonymity of respondents, we use the pronoun 'she' when referring to the interviewee regardless of gender.

\section{RESULTS}

Overall, respondents recognised the new opportunities and potential benefits offered by EUCS. However, most were hesitant about implementing this type of (population-wide) screening in the health sector. Some were in favour of EUCS, provided certain conditions of due care could be met. 


\section{Advantage 1: EUCS maximises opportunities for autonomous} reproductive choice

Respondents were critical about the potential of EUCS for maximising opportunities for autonomous reproductive choice. Their main concerns were whether EUCS will enable prospective parents to make (a) meaningful, (b) informed and (c) free choices.

(a) Are EUCS choices regarded as meaningful? One of the remarks was that in the case of EUCS, the demand for screening does not come from the population itself; it is, on the contrary, a top-down initiative prompted by the health care system. Respondents indicated that in this regard, EUCS distinguishes itself from most ancestry-based screening programmes, which often stem from a community's experience with the suffering of families who are struck by a high burden of disease. The main problem of the top-down nature of EUCS was that, according to many respondents, the majority of the population does not seem to consider carrier screening to be meaningful; a midwife said she was prepared to offer screening, provided that people indicate that they are in need of it' (\#1). As respondents had the impression that most people do not to recognise carrier screening as responding to an urgent problem, many of them were cautious and reserved about introducing EUCS. As one respondent put it:

'... the whole push to introduce carrier screening actually comes from a relatively small group of researchers who continuously talk about it, but cannot convince the rest of the population. [...] It would make a difference if the population felt a sense of urgency that this is a big problem that we should overcome together.' (\#16)

Other respondents, however, argued that the lack of interest in carrier screening does not imply that screening is regarded as unimportant: it merely shows that many people are not aware of the risk of being a carrier; the screening offer is not deliberately rejected, but simply because people 'don't know about it' (\#10). By offering screening to the whole population, individuals are given the opportunity to decide for themselves whether or not to be informed about their carrier status. One respondent called it 'paternalistic' and 'unethical' to deprive people from this information:

'... if I would be pregnant and give birth to a diseased child, and afterwards, I would hear about it [carrier screening], I would feel a bit cheated. So, personally, I think it is unethical not to do it [offer information about carrier screening].' (\#2)

(b) Will people still be able to make informed choices? A different concern expressed by some respondents related to the quantity and diversity of disorders that will be included in the EUCS panel. In the case of ancestry-based screening, it is already a challenge to provide all couples with the information required to make a well-informed choice, even though the disorders are well known in these groups. The relationship between health and genetics is very complex and some people lack the 'basic knowledge' to understand what it means to be a carrier. In the case of EUCS, it becomes even more complicated to inform people, as couples are generally not familiar with the diseases included in the screening programme. Moreover, respondents wondered whether (prospective) parents will be capable of making informed decisions if the screening panel contains such a large, and perhaps also heterogeneous, group of conditions:

'... the more you offer to parents, the bigger the chance that an autonomous choice becomes impossible. After all, if you don't understand it, how to choose what you are choosing for? So that's rather difficult.' (\#4)

c) Will people still be able to make free choices? As regards the freedom of reproductive choices, respondents were mainly concerned about how societal views on 'reproductive responsibility' will change as a result of EUCS. They expressed two main concerns in this respect. The first relates to possible pressure exerted on couples to partake in the screening programme; will it still be possible for couples to reject the screening offer? To illustrate this worry, one respondent referred to what she said she had heard about Denmark, where it was 'almost not done' to refuse prenatal screening for Down syndrome (\#16). Another respondent indicated that it should be avoided that we reach a situation in which 'it is no longer socially accepted that people don't participate' in carrier screening programmes (\#6). Respondents worried that if such programmes become 'normalised', it will be increasingly difficult for couples to go against the current.

Second, stakeholders were worried about the possible impact of EUCS on the societal willingness to support families with affected children. If such support decreases, couples may not feel entirely free to make reproductive choices according to their own values and preferences. One respondent was especially worried about the way in which health insurance companies and mortgage lenders will deal with the availability of EUCS; are we creating a future in which carrier couples will personally bear the financial consequences of conceiving an affected child?

'... what will insurance companies do with it [knowledge about a couple's carrier status]? [...] Imagine, you know this [carrier status] in advance [...] and you decide to get pregnant. Will [the insurance companies] subsequently say: 'Yes, you could have known this in advance, you shouldn't have done it [becoming pregnant]?' So, I think, I don't know how society and especially insurance companies will deal with the risks.' (\#13)

\section{Advantage 2: EUCS will provide equity of access to carrier testing} services

A few respondents argued that EUCS might improve access to testing services for people who do not have an a priori increased carrier risk. In this population, the birth of an affected child typically comes as a surprise. EUCS will raise awareness of risk factors 'related to the combination of the parents' (\#11). However, respondents also indicated that EUCS might not be the best way to reach high-risk populations. For these populations, ancestry-based screening might be more beneficial, as the offer is adapted to the specific needs of its members. As one respondent put it:

'I think that the advantage of [ancestry-based screening] is that it is possibly more recognisable to the target group, because they often know more about the included conditions.' (\#4)

In order to secure that the needs of those who because of their higher risk stand to benefit most from screening will not be lost from sight, some respondents maintained that EUCS must not replace ancestry-based screening. Special concerns were expressed with regard to people at risk for sickle cell disease; they were said to see it as 'the task of the physician to offer them [carrier screening] if it is in their interest' (\#3). Respondents feared that, with EUCS as a substitute for ancestry-based screening, people belonging to this higher risk 
population would no longer feel themselves specifically addressed by a screening offer made to all other (prospective) parents as well.

\section{Advantage 3: EUCS will reduce the risk of stigmatisation}

Most stakeholders saw a positive connection between EUCS and reducing the risk of stigmatisation. In explaining the role of EUCS in avoiding 'social stigma', they especially welcomed the fact that EUCS does not single out particular ethnic groups as being eligible for screening, but implies a universal screening offer (ie, the ' $U$ ' of EUCS):

'The disadvantage [of ancestry-based screening] could be that it radiates something like stigmatisation in the sense that [...] how do I reveal that I originate from a certain group [...]. So then it could be an advantage to offer it [carrier screening] universally and then you could prevent that people feel stigmatised.' (\#4)

The contribution of EUCS in avoiding 'self-stigma' was mainly related to its expanded screening panels (ie, the 'E' of EUCS). By offering screening for a wide range of diseases, EUCS will identify most, or perhaps even all, individuals as carriers of one or a few genetic disorders. It was thought that in this way, the label 'positive carrier status' could be freed from the image of something that is scary and exceptional:

'... in principle, being a carrier isn't bad. That should also be part of the message: being a carrier is normal, we are all carriers of something. Without carriers, [...] there wouldn't be anyone left.' (\#6)

Some respondents, however, suggested that there might be more efficient ways to avoid stigmatisation than by moving from ancestrybased screening to EUCS, for instance by informing people about the common nature of being a carrier, and by drawing attention to its possible advantages (eg, increased resistance to malaria among sickle cell carriers).

Only a few respondents were more hesitant about the role of EUCS in reducing stigmatisation. Some of them suggested that, while EUCS has the potential to decrease the stigmatisation of specific ethnic groups, it threatens to stigmatise or discriminate people because of their disability. Respondents were concerned that autosomal recessive conditions will be increasingly seen as preventable diseases. They indicated that this connotation will be especially strong if the screening programme consists of a predefined list of disorders. In discussing the types of disorders that should be included in the screening panel, one respondent clearly expressed her aversion to speaking about genetic diseases in terms of gradations of severity:

'I really don't want to make any statement in that direction, because I really think [...] that you put diseases on a kind of scale: 'What [sic] is allowed to be born and what [sic] isn't?' Actually, I don't want to say anything about that.' (\#9)

\section{DISCUSSION}

Advocates of EUCS present this new proposition as a revolutionary step in the history of carrier screening with clear positive effects for (prospective) parents. Our findings nevertheless indicate that the advantages of EUCS may not be as straightforward as is sometimes suggested. The health (policy) professionals and representatives of patient organisations involved in this study acknowledged the potential benefits of EUCS. Their concerns nevertheless show that there is something at stake here; if certain issues do not receive proper attention, EUCS might do more harm than good.
First of all, our data suggest that it should not be taken for granted that people without an a priori increased carrier risk are open to the idea of EUCS. Respondents had different explanations for the presumed reticence towards carrier screening among the general population. In the Netherlands, carrier screening is not common practice besides a few local initiatives. Some respondents claimed that the majority of the population does not seem to recognise carrier screening as responding to an urgent problem. Others maintained that most people are simply unaware of their risk of being a carrier and thus may not be responsive to an offer of screening. It is important to keep in mind that by definition, all screening programmes have a 'topdown' character in the sense of entailing an unsolicited offer that does not respond to an individual request. It would seem that in order to obtain a better understanding of people's attitudes towards EUCS, more attitude and pilot studies are needed. Raising awareness about carrier risk and the possibilities for testing will help people to make a personal, well-considered choice as to whether or not to participate in screening. Thereby, it is important that people realise that, although individually rare, it is estimated that $1-2$ in 100 couples are at risk of having a child affected with a recessively inherited disorder. ${ }^{15}$ This implies that the risk of being a carrier couple is in the same order of magnitude as having a child with Down syndrome at the age of $37 .^{23}$

Our findings also indicate that the provision of more genetic risk information does not automatically translate into more opportunities for meaningful reproductive choice. The challenge of how to secure informed choice in situations where people risk being overwhelmed with genetic information has already been addressed by Elias and Annas in the 1990s. They proposed a new strategy for consent for genetic screening, which they referred to as 'generic consent'. ${ }^{24}$ This strategy does not give a full explanation of the clinical and test characteristics of each condition, but broadly describes the types of conditions being screened for. ${ }^{7}$ The concept of generic consent emphasises that too much information may undermine the process of informed decision-making as much as too little information. Yet, to avoid ending up with what is in fact 'uninformed consent', ${ }^{25}$ quite some information must still be shared. The counsellor needs to address various health-related issues, for instance concerning treatment options, life expectancy and quality of life. Moreover, in line with guidelines for responsible screening, the counsellor should encourage couples to seriously consider the psychological and ethical implications of being tested; truly informed decision-making implies that couples are prepared for the potential emotional impact of a positive test result, and the complex choices they may face in such a case.

The concerns expressed by stakeholders further suggest that the question of whether EUCS will increase the autonomy of prospective parents also depends on the impact of the screening offer on societal views on 'reproductive responsibility'. If society becomes less 'disability-friendly', carrier couples may not feel entirely free to 'make decisions that they judge to be right for them in the circumstances in which they find themselves at the time'. ${ }^{13}$ As a result, screening programmes directed at increasing the reproductive autonomy of prospective parents might unintentionally support an ethos of prevention.

Stakeholders mentioned that EUCS might hinder rather than promote equity of access to testing services: while being potentially beneficial to those without an a priori increased carrier risk, the specific needs of people belonging to particular high-risk groups might be lost from sight. Various stakeholders expressed the view that, if the health care system decides to implement EUCS, it should not replace ancestry-based screening. The importance of offering ancestry-based 
screening alongside EUCS has only been recently addressed in the literature. ${ }^{15}$ The general expectation appears to be that the introduction of EUCS panels will make ancestry-based panels obsolete. For instance, Edwards et al ${ }^{7}$ suggest that 'expanded carrier screening can provide information about carrier status beyond population estimates and eliminates the need for ancestry-based screening'.

Respondents saw a positive connection between EUCS and reducing stigmatisation. However, most did not consider this a separate argument for introducing EUCS, but rather as an additional benefit in case such screening was justified on further grounds. Our findings also call attention to a possible downside of EUCS as regards stigmatisation: while having the potential to reduce ethnicity-based stigmatisation, it might reinforce disability-based stigmatisation. This concern has also been expressed by Harper and Clarke, ${ }^{26}$ who argue that 'a carrier screening programme could even exacerbate the situation by making society less tolerant of affected individuals and their parents'.

So far, a few studies have explored the attitudes of US health care providers with regard to EUCS. ${ }^{12,27,28}$ To our knowledge, this is the first study examining the attitudes of health (policy) professionals and representatives of patient organisations within a European context. Moreover, contrary to previous research, this study explicitly focusses on the ethical dimension of EUCS rather than on practical implementation challenges. Several limitations were associated with this study. Since EUCS is not widely available to date, respondents may have had limited familiarity with it. This nevertheless seems inevitable, as it was the very aim of our study to proactively explore the potential pros and cons of EUCS. Another potential limitation is the comparatively small number of participants involved in our study. The variety of arguments put forward by respondents, however, provided a rich basis for our research. Finally, respondents were not explicitly invited to express their views on the three supposed moral advantages of EUCS. Inclusion of this topic in the questionnaire might have led to more detailed reflections and additional insights. It is nonetheless interesting that respondents questioned these advantages without having been specifically asked for it.

In conclusion, our empirical ethics study suggests that the desirability of EUCS is far from obvious: while having the potential to solve some of the moral challenges emerging from traditional carrier screening, EUCS will likely give rise to a range of new challenges. More research is needed to detect pitfalls and (further) anticipate the moral and practical consequences of this new form of carrier screening.

\section{CONFLICT OF INTEREST}

$\mathrm{KH}$ and $\mathrm{LH}$ are affiliated to a University Hospital that offers expanded carrier screening. The remaining authors declare no conflict of interest.

\section{ACKNOWLEDGEMENTS}

We thank the stakeholders for participating in this study, and Evelien Vos (EV) for conducting part of the stakeholder interviews. The study was funded by the Netherlands Organisation for Health Research and Development (ZonMw grant no. 209040001)
1 Lakeman P, Plass AMC, Henneman L, Bezemer PD, Cornel MC, Ten Kate LP: Preconceptional ancestry-based carrier couple screening for cystic fibrosis and haemoglobinopathies: what determines the intention to participate or not and actual participation? Eur J Hum Genet 2009; 17: 999-1009.

2 Nazareth SB, Lazarin GA, Goldberg JD: Changing trends for genetic carrier screening in the United States. Prenat Diag 2015; 35: 931-935.

3 Cousens NE, Gaff CL, Metcalfe SA et al: Carrier screening for Beta-thalassaemia: a review of international practice. Eur J Hum Gen 2010; 18: 1077-1083.

4 Kaback MM: Population-based genetic screening for reproductive counseling: the Tay Sachs disease model. Eur J Pediatr 2000; 159(Suppl 3): S192-S195.

5 The American College of Obstetrics and Gynaecologists' Committee on Genetics: ACOG Committee Opinion no. 486. Update on Carrier Screening for Cystic Fibrosis. Obstet Gynecol 2011; 117: 1028-1031.

6 De Wert G, Dondorp WJ, Knoppers BM: Preconception care and genetic risk: ethical issues. J Community Genet 2012; 3: 221-228.

7 Edwards JG, Feldman G, Goldberg J et al: Expanded carrier screening in reproductive medicine-points to consider. Obstet Gynecol 2015; 125: 653-662.

8 Lazarin GA, Hawthorne F, Collins NS, Platt EA, Evans EA, Haque IS: Systematic classification of disease severity for evaluation of expanded carrier screening panels. PLoS One 2014; 9: 1-16.

9 Grody WW, Thompson BH, Gregg AR et al: ACMG position statement on prenatal/ preconception expanded carrier screening. Genet Med 2013; 15: 482-483.

10 Langlois S, Benn P, Wilkings-Haug L: Current controversies in prenatal diagnosis 4: pre-conception expanded carrier screening should replace all current prenatal screening for specific single gene disorders. Prenatal Diag 2015; 35: 23-28.

11 Borry P, Henneman L, Lakeman P, Ten Kate LP, Cornel MC, Howard HC: Preconception genetic carrier testing and the commercial offer directly-to- consumers. Hum Reprod 2011; 26: 972-977.

12 Cho D, McGowan ML, Metcalfe J, Sharp RR: Expanded carrier screening in reproductive healthcare: perspectives from genetics professionals. Hum Reprod 2013; 28: 1725-1730.

13 Human Genetics Commission. Increasing options, informing choice: a report on preconception genetic testing and screening. 2011. Available at http://f.hypotheses. org/wp-content/blogs.dir/257/files/2011/04/2011.HGC_.-Increasing-options-informingchoice-final2.pdf.

14 Lazarin GA, Haque IS: Expanded carrier screening: a review of early implementation and literature. Semin Perinatol 2016; 40: 29-34.

15 Henneman L, Borry $P$, Chokoshvili $D$ et al: Responsible implementation of expanded carrier screening. Eur J Hum Gen 2016; 3: e1-e12.

16 Markel H: The stigma of disease: Implications of genetic screening. Am J Med 1992; 93: 209-215.

17 Lakeman P, Plass AM, Henneman L, Bezemer PD, Cornel MC, Ten Kate LP: Threemonth follow-up of Western and non-Western participants in a study on preconceptional ancestry-based carrier couple screening for cystic fibrosis and hemoglobinopathies in the Netherlands. Genet Med 2008; 10: 820-830.

18 Gordon C, Walpole I, Zubrick SR, Bower C: Population screening for cystic fibrosis: knowledge and emotional consequences 18 months later. Am J Med Genet 2003; 120A: 199-208.

19 Axworthy D, Marteau TM, Brock DJH, Bobrow M: Psychological impact of populationbased carrier testing for cystic fibrosis: 3-year follow-up. Lancet 1996; 347: 1443-1446.

20 Ioannou L, Massie J, Collins V, McClaren B, Delatycki MB: Population-based genetic screening for cystic fibrosis: attitudes and outcomes. Public Health Genomi 2010; 13: 449-456.

21 Bell CJ, Dinwiddie DL, Miller NA et al: Carrier testing for severe childhood recessive diseases by next-generation sequencing. Sci Trans/ Med 2011; 3: 65ra4.

22 Pope C, Ziebland S, Mays N: Qualitative research in health care. Analysing qualitative data. BMJ 2000; 320: 114-116.

23 Ropers HH: On the future of genetic risk assessment. J Community Genet 2012; 3: 229-236.

24 Elias S, Annas GJ: Generic consent for genetic screening. New Eng J Med 1994; 330: 1611-1613.

25 Dondorp WJ, Sikkema-Raddatz B, De Die-Smulders D, De Wert G: Arrays in postnatal and prenatal diagnosis: an exploration of the ethics of consent. Hum Mutat 2012; 33: 916-922.

26 Harper PS, Clarke AJ: Genetics Society and Clinical Practice. Oxford, UK: BIOS Scientific Publishers, 1997.

27 Ready K, Haque IS, Srinivasan BS, Marshall JR: Knowledge and attitudes regarding expanded genetic carrier screening among women's healthcare providers. Fert Ster 2012; 97: 407-413.

28 McGowan ML, Cho D, Sharp RR: The changing landscape of carrier screening: expanding technology and options? Health Matrix 2013; 23: 15-33. 